\title{
Workflow Management in Intelligent ERP at Aseman Airlines Company
}

\author{
Hojatollah Rashidi Alashty ${ }^{1}$, Filimonov Aleksandr Borisovich ${ }^{2}$ \\ ${ }^{1}$ Cybernetics Department of the MATI University - Russian State Technological University named after K. E. Tsiolkovsky and member of \\ research group of the Islamic Azad University, Qaemshahr, Mazandaran, Islamic Republic of Iran (Department of management) \\ ${ }^{2}$ Cybernetics Department of the MATI University- Russian State Technological University named after K. E. Tsiolkovsky
}

Email address:

rsh_hojat@yahoo.com (H. R. Alashty), filimon_ab@mail.ru (F. A. Borisovich)

\section{To cite this article:}

Hojatollah Rashidi Alashty, Filimonov Aleksandr Borisovich. Workflow Management in Intelligent ERP at Aseman Airlines Company. Automation, Control and Intelligent Systems. Vol. 3, No. 3, 2015, pp. 31-35. doi: 10.11648/j.acis.20150303.11

\begin{abstract}
The real goal of this paper is to study a framework for intelligent ERP in repair \& maintenance at Aseman Airlines Company based upon workflow management. For this purpose, a workflow management system was designed at Aseman Airlines Company by presenting an intelligent model and applying different concepts like genetic algorithm, repair \& maintenance at depot level, social capital and long-life management system. All managerial \& technological aspects were considered in the design of this system in order for upgrading- efficiency and quality of aviation industry services through applying intelligent systems.
\end{abstract}

Keywords: Workflow Management, Intelligent ERP, Aviation Industry

\section{Introduction}

Managers of organizations and departments involved in the maintenance, repair and overhaul of airplanes are obliged to seek new methods and programs for reducing costs and increasing the efficiency because of two reasons. The first reason is related to high levels of man power and consuming parts and total primary investments which are expensive and the second reason is related to extraordinary importance of performance time and other factors like dollar dependency, technical and somehow applicable dependencies from the other. Upon daily-increase entrance of computer into the world of industry and management, all related parts of industries were introduced to this factor accordingly. Of course, modern and complex industries like aviation industry have benefited from these facilities more ethan other industrial groups even beginning from the stage of primary designing of flying objects up to stage of manufacturing the autopilot systems.

Recently, it is common to use computer in various fields like repair, maintenance, overhaul, natural testing systems, programming for periodic checks, life-saved parts replace, controlling of repairs without any need to issue of paper notes, light digital tasks instead of thick books, warehousing and relevant fields, wide range of controlling networks, educational \& repairing simulations and most of other common fields. Today repairs and maintenance need something more than wrench and usual physical tools. Rather than searching for new methods, here we intend to introduce relevant repairs and maintenance of them as the underexperimented or tested guidelines.

\section{Workflow Management Model in Intelligent ERP}

\subsection{Applicable Model of Workflow in ERP}

Introduced model of this research is mainly oriented on workflow collaborative environment. Figure 1 illustrates introduced model where tasks and roles are defined for collaboration among members.

Such collaborative environment includes three key concepts as follows: Members, roles and duties. Members are key factors in a collaborative environment including all important members in aviation industry in the fields of control, handling, maintenance, guidance, flight safety, flight engineers, servants and generally all active parts in aviation industry. Information is produced by members and while performing their assignments. For instance, flight safety engineers control and report relevant information of safety 
system of airplane in each flight and supply flight safety in collaboration with other technical units. Members in Aseman Airlines Company may have one or more official roles. For instance, we have Airplane mechanic engineer, airplane maintenance engineer, flight safety engineer, mechanical designer and so on. Furthermore, there are various up-down relations among them. They will provide a hierarchy of duties named as "Duties Tree". Duties tree illustrates the architecture of collaborative team of Aseman Airlines Company. In addition to members drawing, duties tree has also duties model drawing. Regarding any relations among members we should point out to the important role of social capital of members in upgrading of services level at this airlines company. For explanation of this conception, which can influence on workflow, assume there are infrastructure capitals in aviation industry like enough hardware and

Collaborative Environment

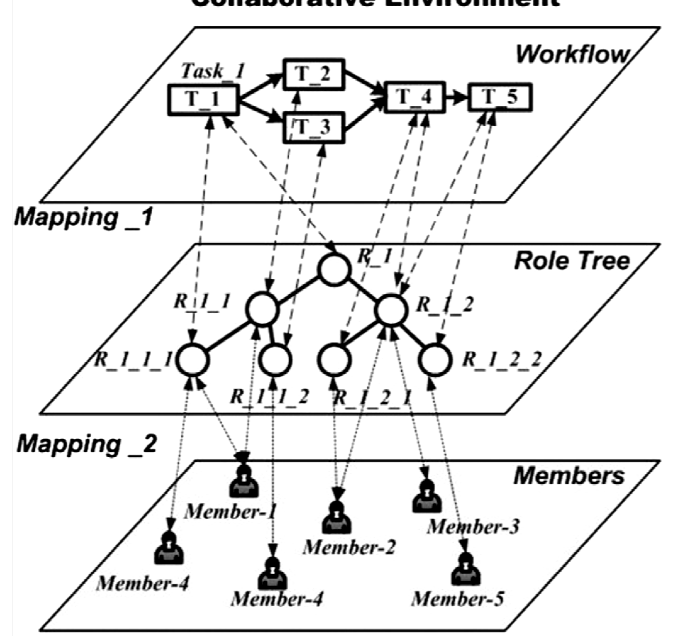

Figure 1. Workflow model based on collaborative environment with definition of tasks and roles.

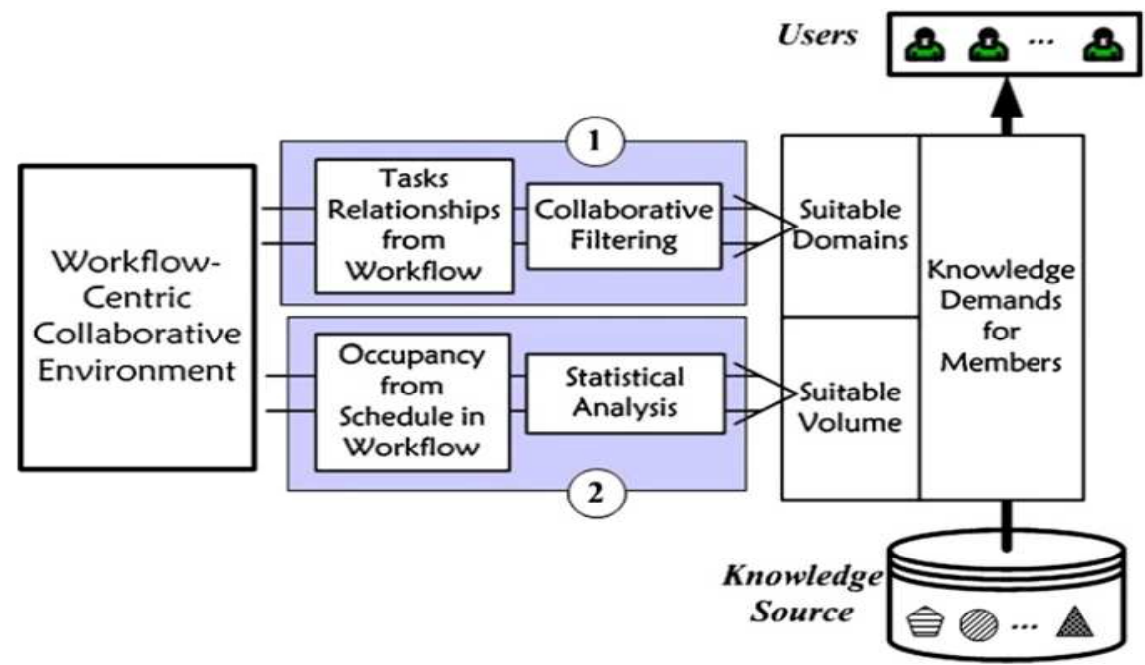

Diagram 1. Work flow \& Members of Airlines Company.
Diagram 1 illustrates major framework of instructing system based upon workflow. There are two kernel modules as specified in this model.

There is a new collaborative filtering for the first problem based upon duty relations (or roles) of work flow in order to software facilities. Also we may assume that all members of company passed enough education and in fact company human capital has a suitable situation. But only mentioned infrastructures and human capital are not enough in improvement of services, but it is necessary to have suitable cooperation among organizational members. They should find suitable cooperation and interaction with each other for organizational goals achievement. Such a cooperation among members is considered as required social capital for achieving better effects of workflow system. Therefore any attention to social capital plays a great role. But benefiting from advanced software systems will assist to more interaction among members and reduce organizational costs and increase quality of services at Aseman Airlines Company.

Diagram 2 illustrates the roles of all members.

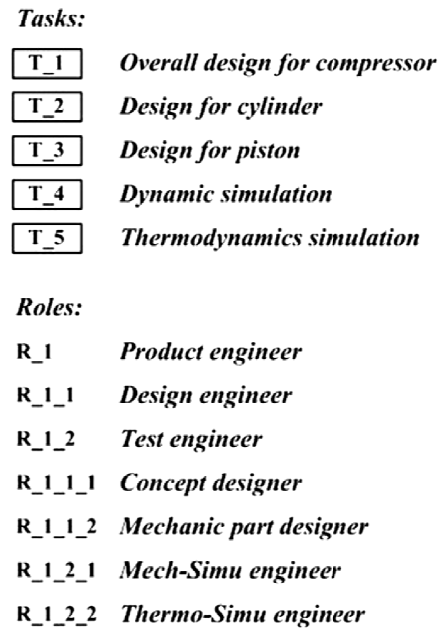


non-harmonized duties into an independent process. There are lots of information in workflow. For instance, we have logical sequential relations among any duties (activities) of team members and resource information of member - roles duties.

It illustrates which member plays what role or carry out what duties. It is possible to combine above-mentioned information with a cooperative filtering in order to find out any requests of members for more information of similar colleagues. It may guarantee that suitable modules of information resources could be recommended to suitable members in a cooperative team [4-6].

There is a fixed analysis method for second problem and finding out involvement rate in proposed workflow for recognition of suitable volume of recommended information [3-5].

Following two parts present partial information about above-mentioned key problems as well.

In second module statistical analysis has a fundamental role for guidance, control and safety of flight. Here we may present a modern analysis of this module:

One of the most common and oldest methods of Optimization Approaches is analytical solving and applying mathematical methods. This is applicable by the use of Objective Function derivation and also current limitations in different issues. Then it is possible to determine maximum or minimum point by the use of second derivative function [2-3]

Regarding any issues in which target function is a little more complex and with hardly derivation, we may use Numerical Approaches. Although it may reduce work accuracy and/or cause non-fixed condition in problem solving, but it may assist to simplify and solve the most engineering problems. Both methods, in complex situations, both mentioned methods need difficult, long-term and bothering calculations with lots of possible mistakes. In addition, these methods may be trapped with local optimizations and do not specify general optimization in alternative functions or Local Optimums and General Optimum.

The most important point is the lack of a special function for defining of problem. Most of mentioned problems are resulted from various functions even with special forms in different conditions of variants. Therefore in such a condition, it is impossible to apply analytical \& numeric methods for any derivation.

For removing such a problem we may apply Genetic Algorithm (GA) as a modern method and a powerful tool in solving complex problems of optimization [7, 8].

Genetic Algorithm is a searching algorithm with a guided search which tries to find a good answer for an assumed optimized function with examination of it. But what makes it different from other methods is parallel and random search. This means that genetic algorithm will test a collection of search spaces in each generation and benefits from transfer and random selection in next generation appointments. Since it may benefit from answer test, it is not necessary to have an derivable function. It will find its optimization in any way. In addition and by random search, algorithm genetic has broken some of complexity bounds. In a clear form and from theoretical viewpoint, if calculation complexity of problem solving need great number of instructions out of the range of facilities in current computer. Then it is possible to solve them by the help of genetic algorithm and quantitative instructions $[7,8]$.

The other point is that functions which are considered in different issues may be discontinuous and non-derivable and non-convex. The mentioned conditions will make it difficult problem or impossible to solve function optimally,. As a result, it is necessary to have a wide range of search in any problems with great solving space. But exact methods have usually high calculation costs. As a result, in spite that random search methods are included in the group of random algorithms, but they are used for solving of geometrical problems. Usually there are acceptable close solutions for practical optimization, because it is preferred to find a close optimized answer for a logical period of time in order find complete optimized solution in a long-term and expensive time $[7,8]$.

\subsection{Encoding in Intelligent Systems Based Upon Genetic Algorithm}

Encoding is one of the major issues in genetic algorithm as mentioned in following encoding methods:

\section{A) Binary Encoding}

It is a common method in which we have genetic algorithm. Variables would be replaced with suitable strings of 0 's and 1 's in this method. The number of bits for encoding of variables depends upon required precise for answers and change limitation of variables. Encoded variables are placed in a sequential string. Bits may be encoded in binary encoding in integers or real numbers [7, 8].

Oliveira and Loucks specified that Real-Value encoding is more effective than binary one. Coding components of possible solutions into a chromosome is the first part of a GA formulation. Each chromosome is a potential solution and is comprised of a series of substrings or genes, representing components or variables that either form or can be used to evaluate the objective function of the problem. [9]

Each position in a chromosome is a real value. Real-value vectors are especially useful for solving real-value optimization problems. Permutations area popular representation for some combinatorial optimization problems [11].

As a result, the strings include all encoded variants in concerned issue for illustrating a point in solution space known as a chromosome. Followings are encoded chromosomes:

Chromosome A: 10110001111010100001110101

Chromosome B: 111110000001010100001101000

There are two types of encoding such as continuous and discrete variables encoding. If the upper and lower bounds of a variable like $x$ are respectively $x_{u}$ and $x_{u}$ and $\boldsymbol{\varepsilon}$ is the accuracy rate of it for binary encoding of continuous variables, the following formula is used for determining a 
minimum number of required bits for variable $[7,8]$.

$$
2^{m} \geq \frac{x_{u}-x_{l}}{\varepsilon}+1
$$

where $\mathrm{m}$ is the minimum number of bits required for the variable.

For binary encoding of discrete variables the length of $\mathrm{m}$ in discrete variables depends upon the number of these variables. For instance, if there are 16 discrete variables we should consider four bits for the string length of each variable. Table 1 illustrates any relation between discrete variables and binary string $[7,8]$.

Table 1. Relation of discrete variables \& binary string.

\begin{tabular}{lcc}
\hline Value of discrete variants & Variable number & Binary string similar to variable \\
\hline$D_{1}$ & 0 & 0000 \\
$D_{2}$ & 1 & 0001 \\
$D_{3}$ & 2 & 0010 \\
$\cdot$ & $\cdot$ & $\cdot$ \\
$\cdot$ & $\cdot$ & $\cdot$ \\
$\cdot$ & $\cdot$ & $\cdot$ \\
$D_{15}$ & 15 & 1111 \\
\hline
\end{tabular}

Excessive-Distribution method is recommended if the number of discrete variables are non-definable by $2^{m}$. There are more than one binary digit would be allocated for every variable in this method $[7,8]$.

B) Permutation encoding

Permutation encoding can be used in ordering problems. In permutation encoding, every chromosome is a string of numbers, which represents number in a sequence as follows:

Chromosome A: 153264798

Chromosome B: 856723149

It is necessary to make some modifications after some combinations and mutations in permutation encoding. This is for ensuring about real sequence in a string $[7,8]$.

The mutation depends on the encoding as well as the crossover. For example when we are encoding permutations, mutation could be exchanging two genes [10].

\section{C) Value Encoding}

Direct value encoding can be used in problems, where some complicated value, such as real numbers, are used. Use of binary encoding for this type of problems would be very difficult. In value encoding, every chromosome is a string of some values. Values can be anything connected to problem, form numbers, real numbers or chars to some complicated objects.

Value encoding is very good and useful for some special problems. On the other hand, for this encoding is often necessary to develop some new crossover and mutation specific for the problem $[7,8]$.

\section{D) Tree Encoding}

Tree encoding is used mainly for evolving programs or expressions, for genetic programming. In tree encoding every chromosome is a tree of some objects, such as functions or commands in programming language.. Tree encoding is good and useful for evolving programs. Programing language LISP is often used for this, because programs in it are represented in this form and can be easily parsed as a tree, so the crossover and mutation can be done relatively easily, like a tree as illustrated in following figure, we may assume input and output values. The target is finding a function with the best outputs for the inputs $[7,8]$.

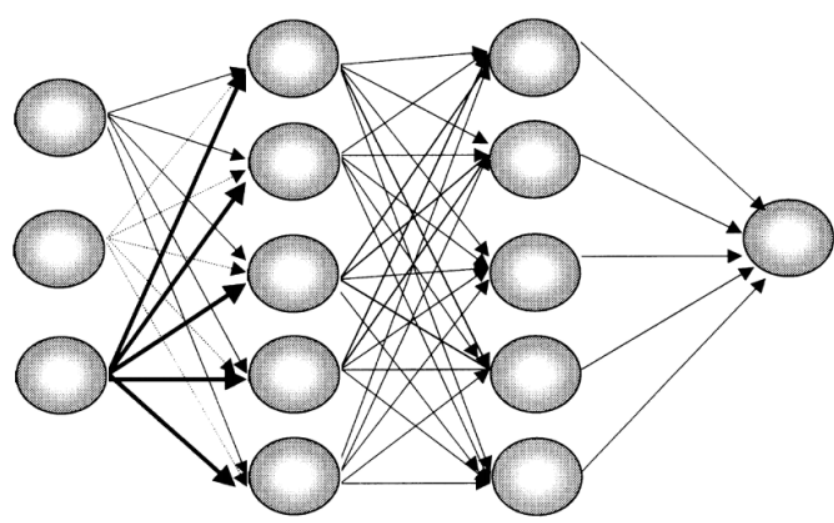

Figure 2. Tree encoding in genetic algorithm.

Finally it is possible to apply genetic algorithms in designing of intelligent ERP systems at Airlines Company for statistical analysis and designing of intelligent control systems as mentioned in this research $[7,8]$.

Furthermore following models are proposed in repair and maintenance of airplanes:

\section{A- Repair \& Maintenance at depot level}

The mentioned Repair \& Maintenance IT could provide required information of equipment according to serial number. After providing of this technology, the system would be enabled for program repair and maintenance at parts level (depot level). Then it is possible to estimate required repairs and maintenance for benefiting from flying through specified times for this purpose. This system activates automatic link of recorded information at flight for prevention from any interfere for persons in charge of repair and maintenance due to very emphatic information. Also it makes it possible to view and evaluate the fleet with more than 750 aircraft and 2000 motors. Also it may browse general view and conditions for approving repair and maintenance management $[1,4]$.

Repair and maintenance information are reserved in a way to enable controlled system works as softness as possible. The mentioned information would be sent to central data base and make it possible to estimate required spare parts (if a network connection is available) in future at fleet and 
making requests from resources through internet. Also it is used for sending repair \& maintenance modifications from central department to the headquarters for daily updates. Now this system is able to send any recorded information about repairs and maintenance of landing airplane from one headquarter to another. Flight technicians prefer this system more because of its friendly and easy usage. It enables all technicians to allocate more times for real repairs and maintenance. Also they should spend little time for preparing of complex reports and filling of forms $[1,4]$.

\section{B: Management system for long-life}

The real goal of any cargo, passenger or military plane is facility of repair, maintenance and its permanence through the long-life. With creation of clear and suitable responsibilities for reaching more confidence and efficiency from ownership time up to the final step with economy, management system for long-life may improve support process as well. Various modifications like end-to-end are originated from non-military airplane facilities. Airplane manufacturers supply a program for supporting of parts, updating, regular documentation, and relevant information of repair and maintenance and information packs as a part of total value of collection $[4,5]$.

\section{Conclusion \& Future Work}

This research is about workflow management system and its application in intelligent ERP systems at aviation industry. The mentioned innovation in this research is introducing and applying advanced genetic algorithm in designing the system and also statistical analysis as a fundament module in workflow system. This results in upgrading repair and maintenance of ERP systems at Aseman Airlines Company. Therefore, it is proposed to apply genetic algorithm and pay more attention to social capital of this process. In addition, this research presents any repair and maintenance at depot level along with long-life management system based upon workflow management at aviation industry. Upon applying of intelligent systems, all managerial and technological aspects of this system have been considered for upgrading of efficiency and quality of services at aviation industry accordingly.

\section{References}

[1] R.H. Sprague, Jr., "A Framework for the Development of Decision Support Systems," MIS Quarterly, 4, no. 4 (December 1980), pp. 1-26.

[2] P. Gray and H.J. Watson, Decision Support in the Data Warehouse, Upper Saddle River, NJ, Prentice-Hall, 1998.

[3] C. White, "Now is the Time for Real-Time BI," DM Review, (September 2004), pp. 47-54.

[4] Richard Hackathorn, "The BI Watch: Real-Time to Real Value," DM Review, 14, no. 1 (January 2004).

[5] Additional information about the real-time BI best practices at Continental is described in R. Anderson-Lehman, H.J. Watson, B.H. Wixom, and J.A. Hoffer, "Continental Airlines Flies High with Real-Time Business Intelligence," MIS Quarterly Executive, 3, no. 4 (December 2004), pp. 163-176.

[6] The story of Continental's turnaround can be found in G. Brenneman, "Right Away and All at Once: How We Saved Continental," Harvard Business Review, 76, no. 5 (September/October 1998), pp. 162-74.

[7] Syswerda, G. (1989): "Uniform Crossover in Genetic Algorithms, In: Proceedings of the Third International Conference on Genetic Algorithms", Schaffer, J. (Ed), Morgan Kauman Publishers, Los Altos, CA, PP. 2-9.

[8] Mitchell, M. (1995): "Genetic Algorithms: An Overview", Cambridge, Mass Complexity, 1(1), PP. 31-39.

[9] Oliveira, R., and Loucks, D. P. (1997). "Operating rules for multireservoir systems.” Water Resour. Res., 33(4), PP. 839852.

[10] ZHANG. J, Chung. H and Lo. W. L, "Clustering-Based Adaptive Crossover and Mutation Probabilities for Genetic Algorithms", IEEE Transactions on Evolutionary Computation vol.11, no.3, pp. 326-335, 2007.

[11] J. D. Schaffer, "A study of control parameters affecting on-line performance of genetic algorithms for function optimization," in Proceedings of the 3rd International Conference on Genetic Algorithms, pp.675-682, 1989. 